\title{
A study of the key genes and inflammatory signaling pathways involved in HLA-B27-associated acute anterior uveitis families
}

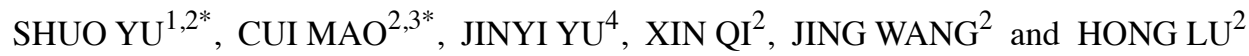 \\ ${ }^{1}$ Department of Ophthalmology, Peking University Third Hospital, Beijing 100083; ${ }^{2}$ Department of Ophthalmology, \\ Beijing Chao-Yang Hospital, Capital Medical University, Beijing 100020; ${ }^{3}$ Department of Ophthalmology, \\ The Third Affiliated Hospital of Chongqing Medical University, Chongqing, Sichuan 400010; \\ ${ }^{4}$ Department of Ophthalmology, Yantai Yuhuangding Hospital, Affiliated Hospital of Medical College, \\ Qingdao University, Yantai, Shandong 264000, P.R. China
}

Received November 6, 2016; Accepted February 21, 2018

DOI: $10.3892 / \mathrm{ijmm} .2018 .3596$

\begin{abstract}
The present study was conducted to investigate the key genes and the inflammatory signaling pathways involved in HLA-B27-associated acute anterior uveitis (AAU) families. Four families with HLA-B27-positive AAU patients and their HLA-B27-positive blood relatives were included in the study. Peripheral blood monocytes were isolated from the subjects and stimulated by lipopolysaccharides (LPS). Gene expression microarrays were used to identify the differentially expressed genes (DEGs), and the DEGs were analyzed by a range of bioinformatics-based techniques, including Gene Ontology (GO), Pathway analysis, Signal-Net analysis and Gene Relation Network (Gene-Rel-Net). Finally, ELISA was used to quantify cytokines in the supernatant. The gene expression microarrays identified 801 DEGs, including 349 upregulated and 452 downregulated genes. The GO analysis revealed several important functions, including metabolic, immune and inflammatory responses. The pathway analysis highlighted the enhanced activity of Staphylococcus aureus infection, chemokine and metabolic signaling pathways, as well as cytokine-to-cytokine receptor interactions. A total of 18 DEGs that were found to play critical roles by Signal-Net and Gene-Rel-Net and verified by quantitative polymerase chain reaction analysis were identified as key genes. In conclusion, monocytes from the AUU patients were more sensitive and exhibited a more prominent inflammatory response to stimulation by LPS compared with monocytes from healthy HLA-B27-positive blood relatives. These characterized DEGs
\end{abstract}

Correspondence to: Professor Hong Lu, Department of Ophthalmology, Beijing Chao-Yang Hospital, Capital Medical University, 8 Gongren Tiyuchang Nanlu, Beijing 100020, P.R. China

E-mail: honglucmu@outlook.com

${ }^{*}$ Contributed equally

Key words: HLA-B27-associated acute anterior uveitis, differentially expressed genes, signal transduction pathway, gene chip may provide new evidence for the pathogenesis of AAU and help identify new therapeutic targets.

\section{Introduction}

Uveitis, which affects the iris, ciliary body and choroid, is an intraocular inflammatory disease and an important cause of visual impairment, even blindness (1). Based on the anatomical localization of inflammation, uveitis may be classified as anterior, posterior and panuveitis (2). Anterior uveitis (AU) is the most prevalent type of uveitis. HLA-B27-associated acute AU (AAU) was originally described in 1973 and remains the most common type of AAU. The arthrogenic/uveitogenic peptide hypothesis (3) and the HLA-B27 misfolding hypothesis have been proposed to explain the role of HLA-B27 in AAU. There is also evidence that innate immunity may be involved, in addition to the role of adaptive immunity, in the pathogenesis of HLA-B27-related disease. Since elements of both innate and adaptive immune reactions are observed, HLA-B27-related disease should be placed in the spectrum between autoimmune and autoinflammatory disease (4).

The basic concept of arthrogenic/uveitogenic peptide hypothesis is that HLA-B27 molecules present antigens that share sequence homology with self-peptides to HLA-B27-restricted $\mathrm{CD}^{+} \mathrm{T}$ cells, which are autoreactive (5). These activated $\mathrm{T}$ cells induce inflammation as a result of a cross-reactivity with peptides in the eye (3). HLA-B27 heavy chains have a tendency to misfold, and it has been suggested that such misfolding may lead to the development of HLA-B27-related disease $(6,7)$.

Extensive clinical and laboratory research has provided evidence that Gram-negative bacteria and their lipopolysaccharides (LPS) are associated with AAU (8). According to recent studies, the earliest responders to bacterial infection are mononuclear cells (9). Once activated, mononuclear cells produced a series of inflammatory cytokines and chemokines that cause marked amplification of the inflammatory process. Although the pathogenesis is not clear, evidence shows that both adaptive and innate immune responses may be involved in the occurrence of uveitis $(10,11)$. To explore the pathogenetic mechanism of AAU, this study group found prominent 
differences between the monocytes of HLA-B27-positive AAU patients and HLA-B27-negative healthy controls. In response to LPS stimulation, 1,105 and 25 genes were upregulated in the HLA-B27-positive AAU patients and HLA-B27-negative healthy controls, respectively. Gene Ontology (GO) and pathway analysis illustrated that these genes participated in protein transport and folding, which are key to the inflammatory process (12). In the present study, we compared the gene expression profiles of monocytes from HLA-B27 AAU susceptible families before and after LPS stimulation using Affymetrix microarrays. According to Signal-Net, Gene Relation Network (Gene-Rel-Net), GO and Kyoto Encyclopedia of Genes and Genomes (KEGG) analysis, we also demonstrated the role of these different genes in inflammation. Currently, one of the major challenges is to identify key genes involved in HLA-B27 AAU and to design more effective targeted anti-inflammatory therapeutic strategies.

\section{Materials and methods}

Study population. Patients who suffered from HLA-B27associated AAU without systemic immune disease were diagnosed and classified according to the International Uveitis Study Group Standards (13) and were recruited to the Eye Clinic of Beijing Chao-Yang Hospital, between September 2013 and March 2014. Four patients who fulfilled these criteria and their immediate HLA-B27-positive healthy (without AAU or systemic immune disease) family members as controls were included in the study. All the subjects had received flow cytometric HLA-B27 testing at Beijing Chao-Yang Hospital prior to enrollment. All candidates provided written informed consent and the clinical research protocol was approved by the Ethics Committee of Beijing Chao-Yang Hospital, Capital Medical University. The eligible subjects included 3 men and 5 women aged 32-55 years (mean age, 42 years). All the patients were in the convalescence stage, with no signs or symptoms, and were only adminsitered topical eye drops, without systemic immune inhibitors.

Isolation and identification of monocytes. Peripheral blood $(16 \mathrm{ml})$ from HLA-B27-positive AAU patients and healthy blood relatives was collected into heparinized tubes and immediately processed in order to separate peripheral blood mononuclear cells (PBMC) with the Ficoll-Hypaque method. The cell viability was $\geq 95 \%$ as determined with trypan blue staining. Each cell suspension was then distributed to chambers of 12-well culture plates with a mean concentration of $1 \times 10^{6} \mathrm{cells} / \mathrm{ml} / \mathrm{well}$. The plates were incubated in a humidified incubator with $5 \% \mathrm{CO}_{2}$ at $37^{\circ} \mathrm{C}$ for $4 \mathrm{~h}$ to ensure that the monocytes adhered to the plates. Non-adherent cells were removed by washing with Hank's Balanced Salt Solution. The monocytes of HLA-B27-positive AAU patients were randomly divided into two groups: Stimulation group (A2) and non-stimulation group (A1). Similarly, the two groups of monocytes from HLA-B27-positive controls were labeled as stimulation group (B2) and non-stimulation group (B1). LPS (1 $\mu \mathrm{g} / \mathrm{ml}$, final concentration) was added to each stimulation group (A2 and B2) and an equal volume of phosphate-buffered saline was added to the non-stimulation groups (A1 and B1). The monocytes were tested with CD14 immunofluorescence stain.

Enzyme-linked immunosorbent assays (ELISA). Samples of culture supernatants were harvested at different time-points
(4, 8, 12 and $24 \mathrm{~h}$ ) following stimulation with LPS and stored in microtubes at $-80^{\circ} \mathrm{C}$. The levels of tumor necrosis factor (TNF)- $\alpha$, interleukin (IL)-10 and IL-6 in cell culture supernatant samples were measured with a standard quantifiable sandwich enzyme immunoassay. ELISA was performed according to the manufacturer's instructions. Measurements were obtained with an automated microplate reader (Multiskan MK3; Thermo Fisher Scientific, Inc., Waltham, MA, USA) at an optical absorbance value of $450 \mathrm{~nm}$.

Total RNA extraction and microarray assay. Total RNA was extracted from 24-h cultured monocytes by using TRIzol reagent (Invitrogen; Thermo Fisher Scientific, Carlsbad, CA, USA) and an RNeasy mini kit (Qiagen, Valencia, CA, USA) according to the manufacturer's protocol. After verifying the integrity, concentration and purity of RNA, cDNA was generated by using a One-Cycle Target Labeling and Control reagent kit (Affymetrix, Santa Clara, CA, USA). Biotinylated and amplified cRNA was generated from the total RNA samples with a GeneChip IVT labeling kit and then hybridized at $45^{\circ} \mathrm{C}$ to Affymetrix GeneChip Human Gene 1.0 ST arrays (both from Affymetrix) for $16 \mathrm{~h}$. The abovementioned procedure was repeated in triplicate. Data were analyzed by GeneChip operating software.

Data analysis. Gene expression patterns from groups A2, B2, A1 and B1 were analyzed to identify differentially expressed genes. We focused on those DEGs that were acquired following comparison between the A2 and B2 groups, but were not present in those acquired from the A1 and B1 groups, eventually narrowing down the key candidate genes. These candidate DEGs were selected after performing a paired t-test, and the threshold of significance was defined by P-value and false discovery rate (FDR) analysis (14-16). The FDR was calculated to correct the P-value, with a smaller FDR indicating a smaller error in the estimated P-value (17). DEGs were identified to be up- or downregulated with a P-value of $<0.05$. GO analysis was used to analyze the main function of the DEGs. GO separated genes into categories, and gene regulatory networks were discovered based on biological processes, which is the key functional classification of the National Center for Biotechnology Information $(18,19)$. Pathway analysis was applied to uncover a significant pathway of the differential genes according to KEGG. Gene-gene interaction network (Signal-Net) was constructed on the basis of the data of DEGs to show the core genes that played a critical role in this network (20-22). The genes were described as indegree, outdegree, or degree. A higher degree indicated that the genes were more strongly correlated with others, and suggested a more important role in the signaling network. In addition, the number of source genes of a gene is referred to as the indegree of the gene and the number of target genes of a gene is its outdegree. Gene-Rel-Net was built according to the normalized signal intensity of specific expression genes (23). For each pair of genes, we calculated the Pearson's correlation and selected the significant correlation pairs to construct the network to locate core regulatory genes (24). Core regulatory factors connected more adjacent genes and had higher degrees. The regulation difference of each gene was compared in different networks. ABS represents the absolute value of the 
Table I. Top five genes ranked by degree following analysis with Signal-Net.

\begin{tabular}{llcccccc}
\hline Gene symbol & \multicolumn{1}{c}{ Description } & Degree & Indegree & Outdegree & Style & P-value & FDR \\
\hline PHGDH & Phosphoglycerate dehydrogenase & 20 & 20 & 20 & Up & 0.014022 & 1 \\
ALB & Albumin & 17 & 17 & 17 & Down & 0.017775 & 1 \\
HLTF & Helicase-like transcription factor & 13 & 13 & 13 & Up & 0.035549 & 1 \\
RNF130 & Ring finger protein 130 & 11 & 11 & 11 & Down & 0.013715 & 1 \\
SNX2 & Sorting nexin 2 & 11 & 11 & 11 & down & 0.029279 & 1 \\
\hline
\end{tabular}

Degree represents the number of gene-to-gene interactions. Indegree and outdegree represent the numbers of upstream and downstream regulated genes, respectively. FDR, false discovery rate.

difference of the relative degree; a higher ABS indicated that the genes have a more significant regulatory ability and play a more important role in the networks.

Reverse transcription-quantitative polymerase chain reaction (RT-qPCR) analysis. A total of 18 candidate genes were selected to perform RT-qPCR analysis using the same sample of the total RNAs that was isolated using an RNeasy kit (Qiagen) according to the manufacturer's protocol. M-MLV reverse transcriptase (Promega, Madison, WI, USA) was used to synthesize cDNA. qPCR analysis and data collection were performed on the ABI 7500 qPCR system. The raw quantifications were normalized to the $\beta$-actin values for each sample and fold changes are shown as mean \pm standard deviation (SD) of three independent experiments.

Statistical analysis. Each experiment was performed independently at least three times. The results are expressed as mean $\pm \mathrm{SD}$. The differentially expressed genes were analyzed using paired Student's t-test. The RT-qPCR and ELISA results were analyzed with $\mathrm{t}$-test. $\mathrm{P}<0.05$ was considered to indicate statistically significant differences.

\section{Results}

Mononuclear cell separation and identification. The monocytes were tested with CD14 immunofluorescence stain. The isolated mononuclear cells were round, kidney-shaped or irregular, with intact nuclei. The results of the immunofluorescence revealed that the monocytes were positive for the cell membrane marker CD14 (Fig. 1).

Differentially expressed genes. A gene chip study was performed by using the Affymetrix probe data set. Paired Student's t-test was performed to identify genes that were expressed separately and differentially. As a result, genes that had a P-value $<0.05$ were considered to be significantly differentially expressed. Of the 801 differentially expressed genes that were initially identified, 349 were upregulated and 452 downregulated. A large negative logarithm of the P-value (-LgP-value) correlated with greater significance of the GO category. As shown in Fig. 2A, some of the upregulated GOs (up-GOs) included metabolic metabolites (cholesterol, threonine, $\gamma$-aminobutyric acid and glyceraldehyde-3-phosphate), regulation of transcription from RNA polymerase II

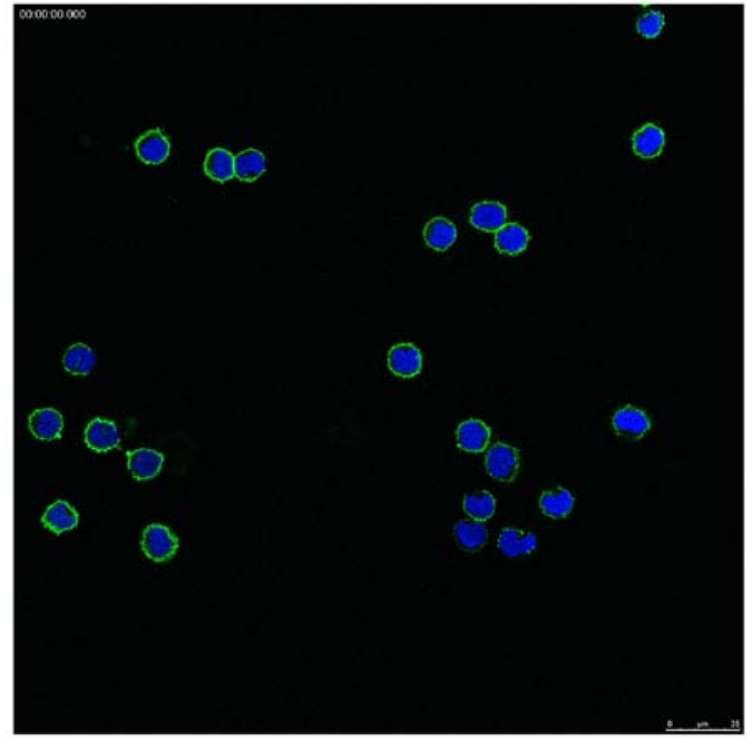

Figure 1. CD14-positive monocytes. Immunofluorescence staining of CD14 (green) was identified on the cell membrane. The nuclei were stained with 4',6-diamidino-2-phenylindole (blue).

promoter, leukocyte chemotaxis involved in inflammatory response, free ubiquitin chain polymerization, LPS transport and mitogen-activated protein kinase (MAPK) import into nucleus. By contrast, some of the top downregulated GOs (down-GOs; Fig. 2B) included immune (adaptive and innate) and inflammatory response, cell communication, positive regulation of I- $\kappa \mathrm{B}$ kinase/nuclear factor (NF)- $\kappa \mathrm{B}$ cascade, signal transduction, small-molecule metabolic processes and transport. Within these GOs, a number of important functions, such as cell communication, NF- $\kappa \mathrm{B}$ cascade, signal transduction, leukocyte chemotaxis and transport, were involved in the regulation of this immune-related disorder. Pathway analysis was used to identify the significant pathway of the differential genes according to KEGG. The Fisher's exact test and $\chi^{2}$ test were used to select the significant pathway, and the threshold of significance was defined by the P-value. The $\mathrm{x}$-axis is the negative logarithm of the P-value. The longer the bar, the smaller the P-value, so that pathway is more significant. As shown in Fig. 3, there were some important pathways, including Staphylococcus aureus infection, chemokine signaling pathway, cytokine-to-cytokine receptor interaction and metabolic pathways. To screen for the important candidate 
A

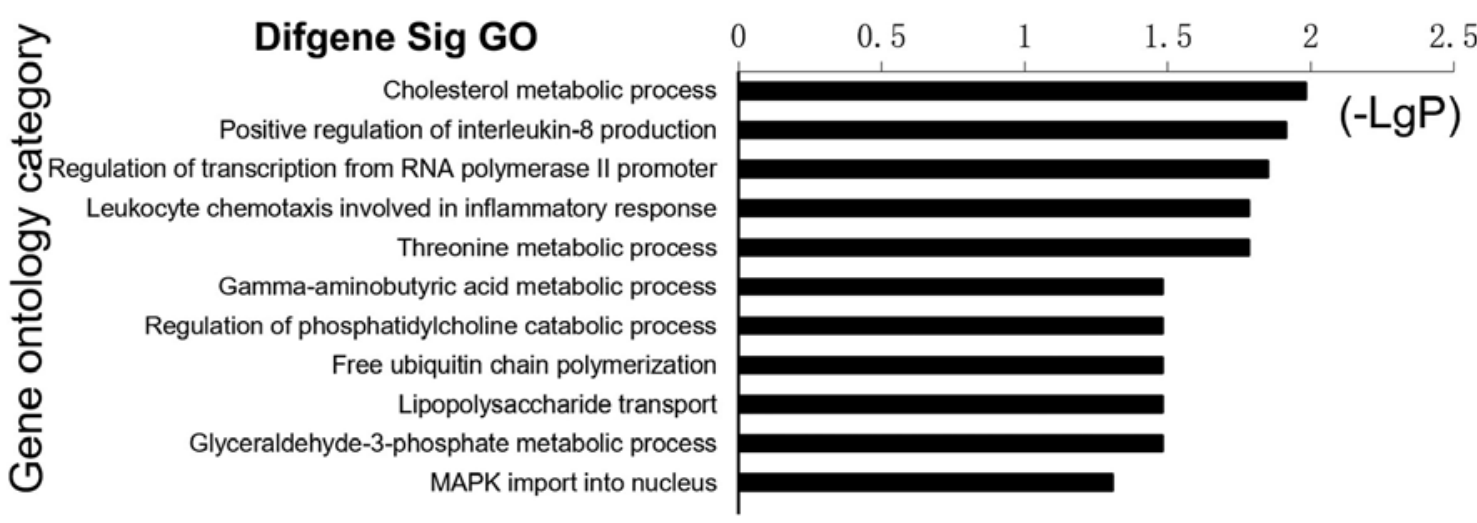

B Difgene Sig GO

Immune response
Inflammatory respons Chemotaxis

Positive regulation of I-kappaB kinase/NF-kappaB cascade Small GTPase mediated signal transduction Response to organic substance Negative regulation of innate immune response Signal transduction Leukocyte chemotaxis Positive regulation of Rho protein signal transduction 0 2

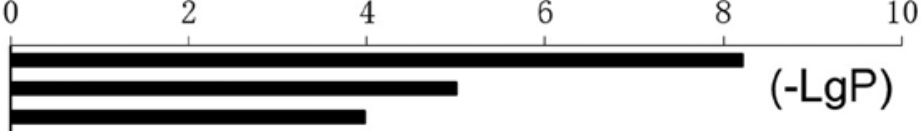
Positive regulation of monocyte chemotaxis
Interleukin-1-mediated signaling pathway Innate immune response Positive regulation of $\mathrm{T}$ cell proliferation Protein autophosphorylation Adaptive immune response Regulation of protein complex disassembly Interleukin-10 secretion Chemokine secretion Positive regulation of GTPase activity Response to stimulus Small molecule metabolic process Negative regulation of $\mathrm{Nf}$-kappaB transcription factor activity Cell-cell signaling Apoptotic process Response to lipopolysaccharide Negative regulation of JNK cascade

Figure 2. Significantly changed Gene Ontologies (GOs) of differentially expressed genes. (A) Up-GOs. (B) Down-GOs. The X-axis shows the negative logarithm of the P-value (-LgP) and the y-axis shows the GO category. A higher-LgP indicated a smaller P-value. A high -LgP-value was correlated with a greater significance of the GO category.

genes among the declared 801 genes, we selected Signal-Net analysis and Gene-Rel-Net, and subsequently identified 18 genes. Signal-Net demonstrated the intensity of association between the differentially expressed genes and adjacent genes. This indicated that the genes were phosphoglycerate dehydrogenase $(P H G D H)$, albumin $(A L B)$, helicase-like transcription factor $(H L T F)$, ring finger protein 130 (RNF130), and sorting nexin 2 (SNX2) with a higher degree (Fig. 4 and Table I). In Gene-Rel-Net, interleukin-1 receptor-associated kinase 3/M (IRAK3/M), microsomal glutathione S-transferase 1 (MGST1), cytochrome b-245, $\beta$ polypeptide $(C Y B B)$, Fc fragment of IgG, high-affinity Ia, receptor (CD64) (FCGR1A), gap junction protein, $\beta 2,26 \mathrm{kDa}(G J B 2)$, chemokine (C-X-C motif) ligand 9 (CXCL9), guanine nucleotide-binding protein (G protein), q polypeptide (GNAQ), cathepsin $\mathrm{S}$ (CTSS), phosphatidic acid phosphatase type 2B (PPAP2B), leukocyte immunoglobulin-like receptor, subfamily $\mathrm{B}$ (with TM and ITIM domains), member 2 (LILRB2), cell division cycle 42 (GTP-binding protein, $25 \mathrm{kDa}$ ) (CDC42), allograft inflammatory factor 1 (AIF1), and fibroblast growth factor 2 (basic) (FGF2) link most adjacent genes and have the largest degrees; thus, they were deemed as the core regulatory factors (genes) (Fig. 5 and Table II).

$R T-q P C R$ verification of the genes. RT-qPCR was used to confirm the expression of the 18 candidate genes. Although the fold changes in mRNA varied between the microarray and RT-qPCR analyses, the expression results were relatively consistent for the analyzed genes. As expected, there were very few differences between the A1 and B1 groups. Compared with the results of the chip between $\mathrm{A} 2$ and $\mathrm{B} 2$, the expression of most genes indicated that the overall trend was consistent with the trend of the chip (Fig. 6).

ELISA. The concentrations of TNF- $\alpha$ (at $24 \mathrm{~h}$ ), IL-6 (at $24 \mathrm{~h}$ ) and IL-10 (at $8 \mathrm{~h}$ ) were compared in the culture supernatant of monocytes isolated from the patients and their blood relatives when the level of the cytokine reached the maximum following 
Table II. Top 13 genes ranked by ABS following analysis with Gene-Rel-Net.

\begin{tabular}{|c|c|c|c|c|c|c|c|}
\hline Gene symbol & Description & Degree A & Degree B & $\begin{array}{c}\mathrm{ABS} \\
(\mathrm{K} 2-\mathrm{K} 1)\end{array}$ & Style & P-value & FDR \\
\hline IRAK3/M & Interleukin-1 receptor-associated kinase 3/M & 30 & 5 & 0.682352941 & Down & 0.028558 & 1 \\
\hline MGST1 & Microsomal glutathione S-transferase 1 & 0 & 17 & 0.68 & Down & 0.044897 & 1 \\
\hline CYBB & Cytochrome $b-245, \beta$ polypeptide & 28 & 4 & 0.663529412 & Down & 0.025561 & 1 \\
\hline FCGR1A & $\begin{array}{l}\text { Fc fragment of IgG, high-affinity Ia, } \\
\text { receptor (CD64) }\end{array}$ & 28 & 4 & 0.663529412 & Down & 0.032623 & 1 \\
\hline GJB2 & Gap junction protein, $\beta 2,26 \mathrm{kDa}$ & 32 & 7 & 0.661176471 & Down & 0.01698 & 1 \\
\hline CXCL9 & Chemokine (C-X-C motif) ligand 9 & 8 & 22 & 0.644705882 & Down & 0.031734 & 1 \\
\hline GNAQ & $\begin{array}{l}\text { Guanine nucleotide-binding protein (G protein), } \\
\text { q polypeptide }\end{array}$ & 5 & 19 & 0.612941176 & Down & 0.012584 & 1 \\
\hline CTSS & Cathepsin $\mathrm{S}$ & 26 & 4 & 0.604705882 & Down & 0.044654 & 1 \\
\hline PPAP2B & Phosphatidic acid phosphatase type 2B & 11 & 21 & 0.516470588 & Down & 0.003548 & 1 \\
\hline LILRB2 & $\begin{array}{l}\text { Leukocyte immunoglobulin-like receptor, } \\
\text { subfamily B (with TM and } \\
\text { ITIM domains), member } 2\end{array}$ & 20 & 2 & 0.508235294 & Down & 0.044337 & 1 \\
\hline $\mathrm{CDC} 42$ & $\begin{array}{l}\text { Cell division cycle } 42 \text { (GTP-binding } \\
\text { protein, } 25 \mathrm{kDa})\end{array}$ & 29 & 9 & 0.492941176 & Down & 0.016927 & 1 \\
\hline AIF1 & Allograft inflammatory factor 1 & 22 & 5 & 0.447058824 & Down & 0.03037 & 1 \\
\hline FGF2 & Fibroblast growth factor 2 (basic) & 22 & 7 & 0.367058824 & $\mathrm{Up}$ & 0.040008 & 1 \\
\hline
\end{tabular}

Degree A and B represent the number of gene-to-gene interactions in groups A and B, respectively. ABS represents the absolute value of the difference of the relative degree. FDR, false discovery rate.

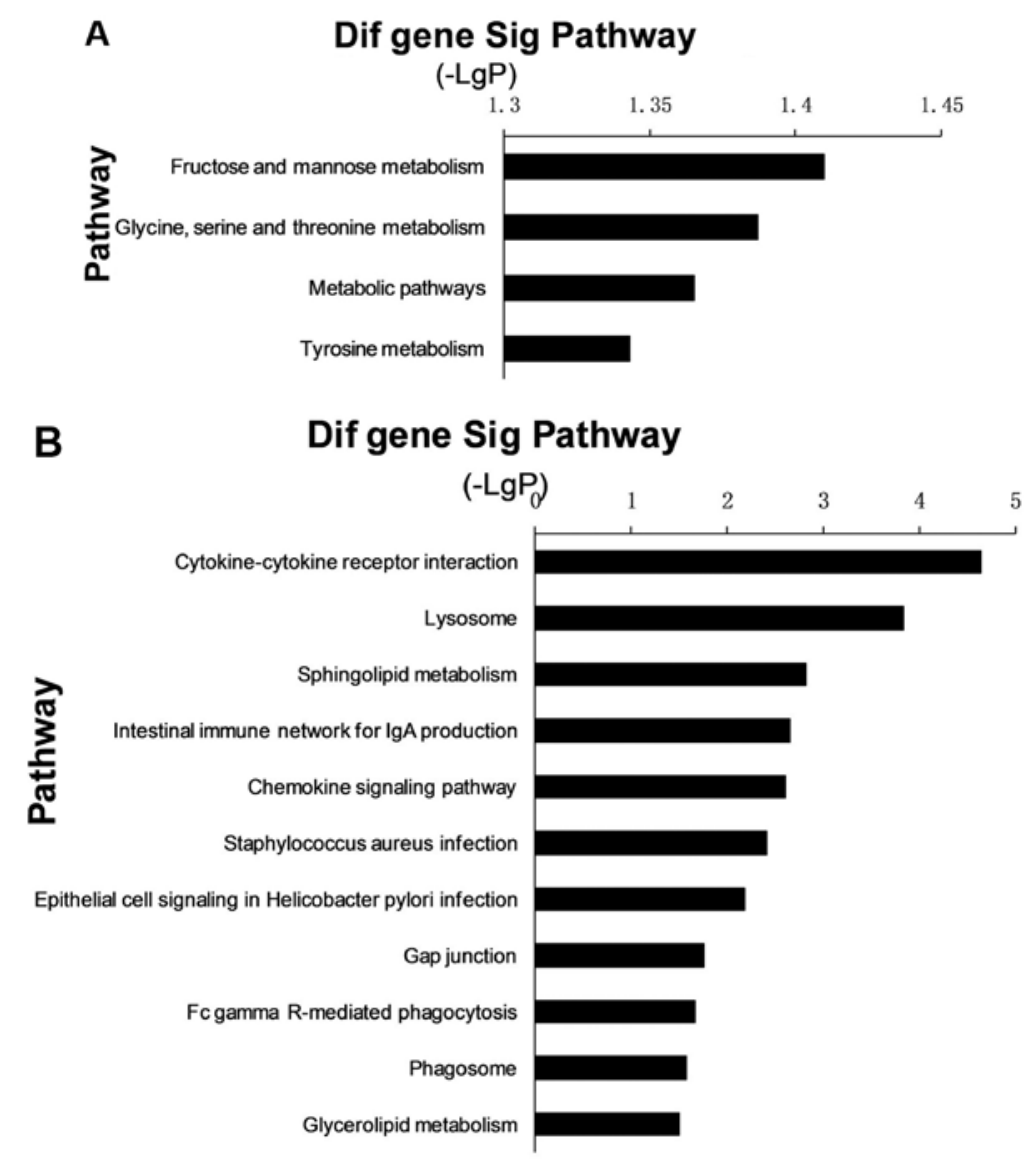

Figure 3. Significant changed pathways based on the KEGG database with P/0.05 and FDR/0.05. (A) Significantly upregulated pathways. (B) Significantly downregulated pathways. The y-axis shows the pathway category and the $\mathrm{x}$-axis shows the negative logarithm of the P-value (-LgP). A higher - $\mathrm{LgP}$ indicated a smaller P-value. KEGG, Kyoto Encyclopedia of Genes and Genomes; FDR, false discovery rate. 


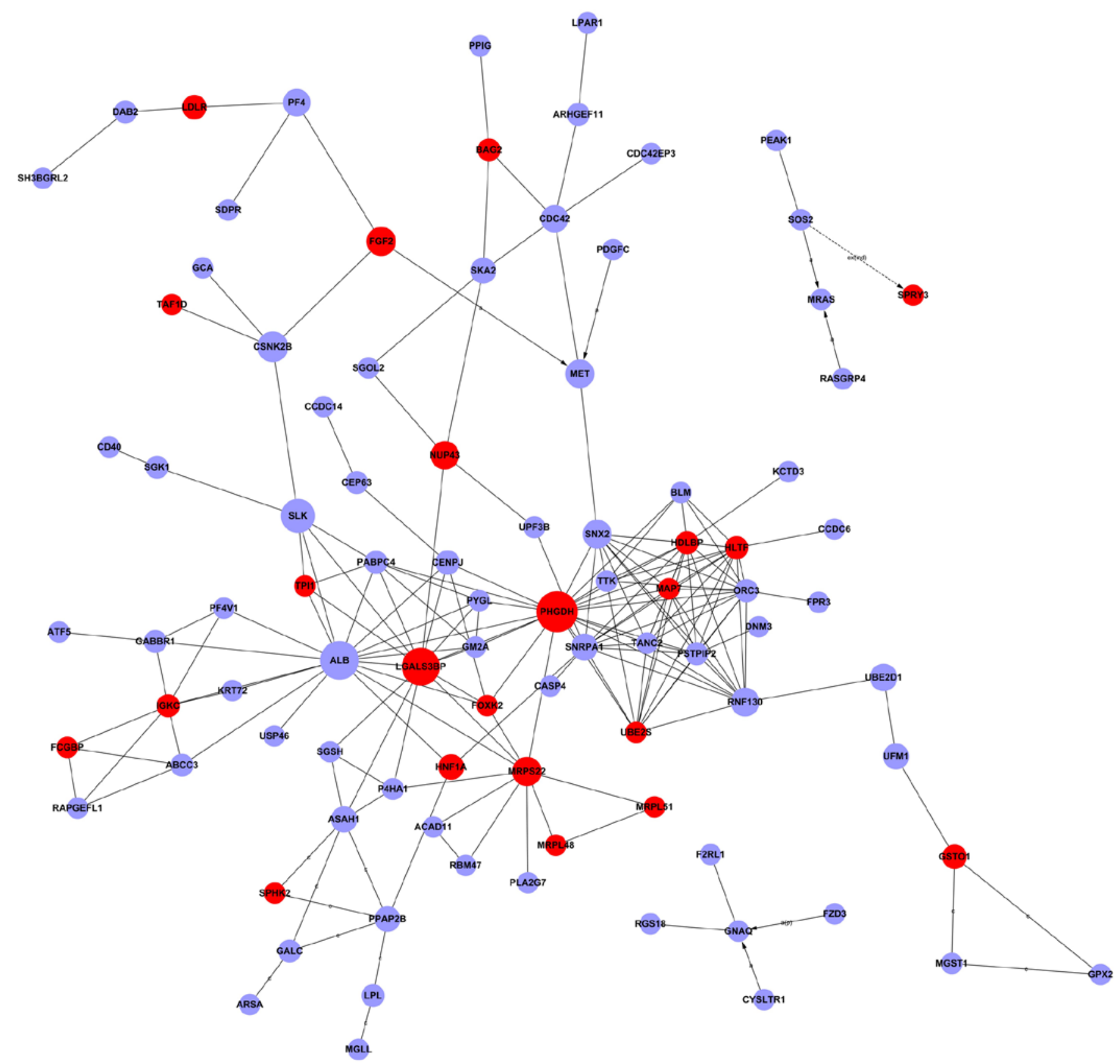

Figure 4. Signal-Net of differentially expressed genes. The red circles represent the upregulated genes and the blue circles represent the downregulated genes. The area covered by the circle represents the degree. Interaction between the genes is shown as: a, activation; a(p), activation (phosphorylation); c, compound; ex(ind), expression (indirect effect).

stimulation with LPS. We found that the concentrations of TNF- $\alpha(118.90 \mathrm{pg} / \mathrm{ml})$ and IL-6 $(91.23 \mathrm{pg} / \mathrm{ml})$ were higher in the patients, while the concentration of IL-10 $(13.35 \mathrm{pg} / \mathrm{ml})$ was higher in their blood relatives (Fig. 7).

\section{Discussion}

HLA-B27 exhibits a wide distribution among geographic regions, which is affecting the prevalence of AAU worldwide. HLA-B27-associated AAU is the most common type of AAU, which represents a potential threat to visual integrity (25); however, the exact underlying mechanism has yet to be determined. Abundant studies have proven that LPS, a major component of Gram-negative bacterial cell walls, can induce
AAU in animal models (26-29). Our previous studies demonstrated that LPS-related Gram-negative bacteria could activate TLR4-mediated immunity, resulting in the development of AAU $(30,31)$. In addition, the extent of inflammation and gene expression levels from monocytes of HLA-B27-positive patients were significantly different compared with normal individuals in response to LPS stimulation (12). Furthermore, $\sim 18-32 \%$ of AAU patients are HLA-B27-positive, but only a few of the HLA-B27 carriers develop AAU (32), as evidenced by HLA-B27-positive patients and blood-related healthy carriers. In the present study, we selected monocytes from patients with HLA-B27-associated AAU and their HLA-B27-positive blood relatives to investigate this anomaly. In order to identify the genes that play more important roles in the development of HLA-B27-associated AAU, we only 


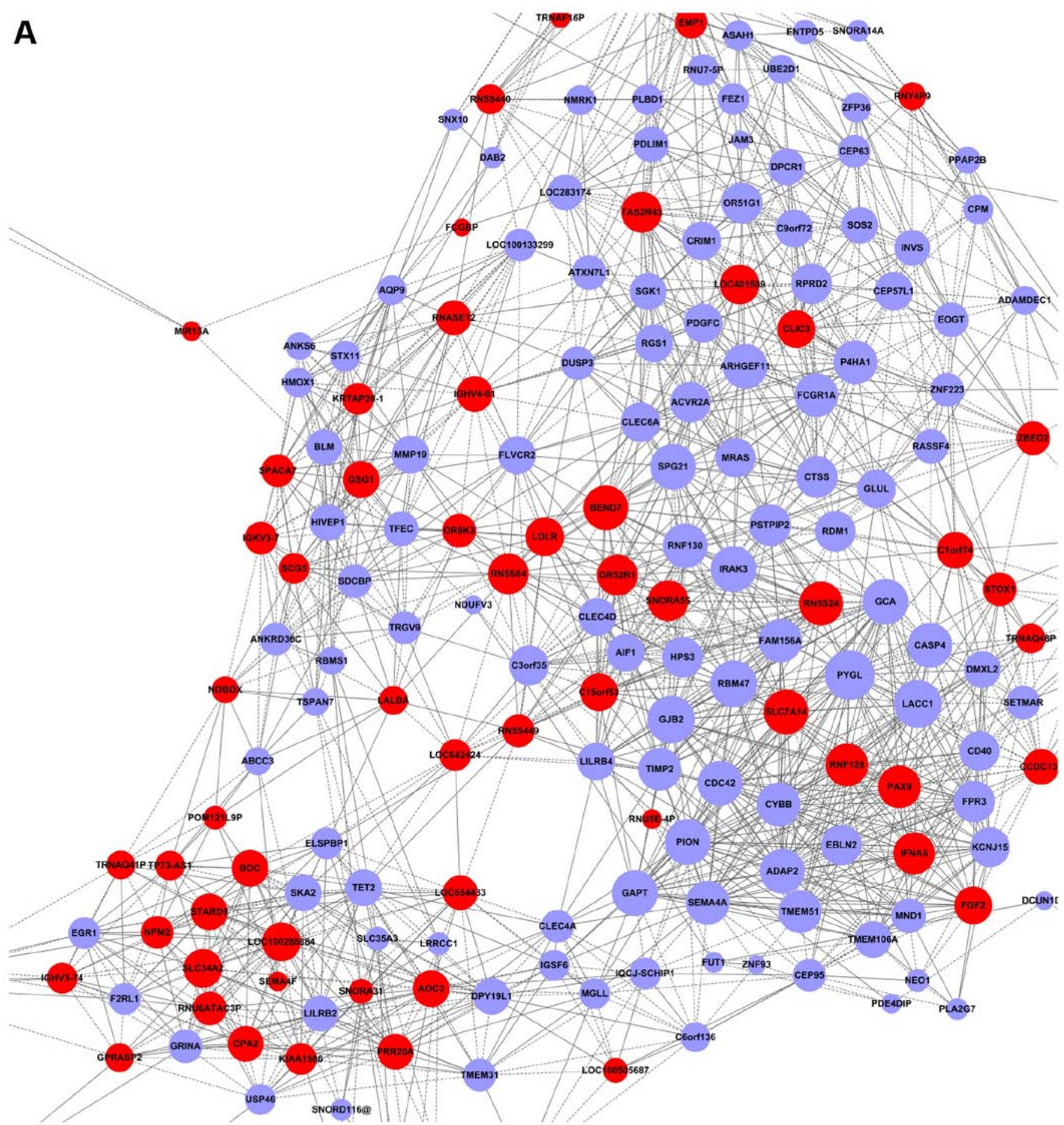

Figure 5. Gene relation network (Gene-Rel-Net) of differentially expressed genes. (A) Differentially expressed genes of HLA-B27-associated acute anterior uveitis.

enrolled HLA-B27-associated AAU patients and their immediate HLA-B27-positive healthy (without AAU or systemic immune disease) family members as controls, which significantly increased the difficulty of the enrollment for this study. Thus, a total of 8 subjects were ultimately enrolled. Although a bigger sample size would potentially optimize the results, the results presented herein exhibited statistical significance. We were also able to identify several potential target genes following data analysis.

Microarray analysis of HLA-B27-associated AAU family has not been reported to date. High-throughout screening is also an effective method for comparing differentially expressed genes between experimental and control groups. In the present study, key genes and inflammatory signaling pathways involved in monocytes of HLA-B27-associated AAU families were studied by gene expression microarray. By comparing gene expression between the two groups, we identified 801 differentially expressed genes, of which 349 were upregulated and 452 were downregulated. The results indicated that LPS stimulation induced an altered expression in monocytes from HLA-B27-positive-AAU patients compared with their blood relatives. The DEGs were subsequently organized into different categories based on the biological processes. The results of 


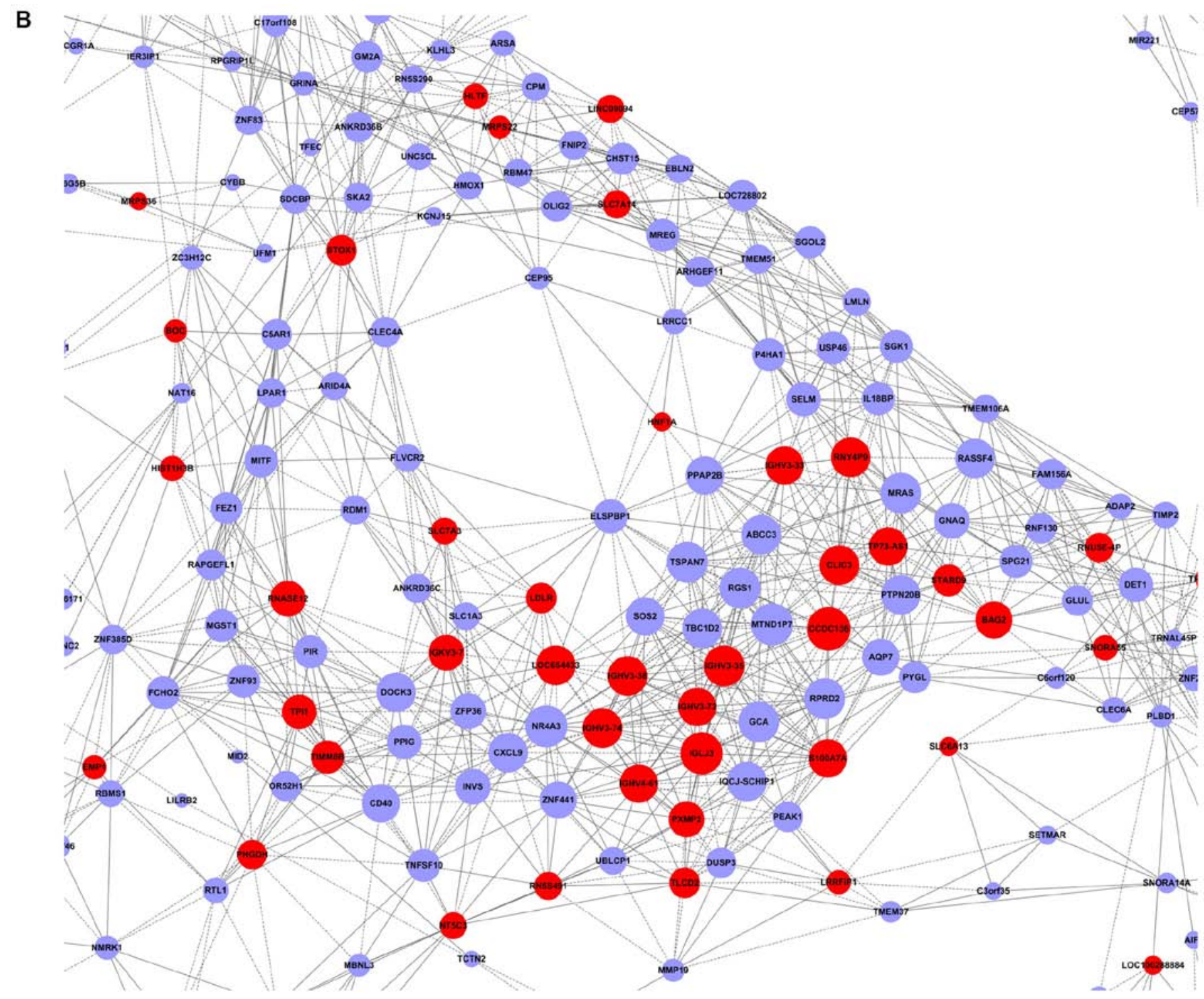

Figure 5. Continued. (B) Differentially expressed genes of HLA-B27-associated controls. The red circles represent the upregulated genes and the blue circles represent the downregulated genes. The area covered by the circle represents the degree.

the GO analysis suggested that immune and inflammatory response, regulation of $\mathrm{I}-\kappa \mathrm{B}$ kinase/NF- $\mathrm{KB}$ cascade, free ubiquitin chain polymerization, MAPK import into nucleus, cytokines, chemokines, as well as small-molecule metabolic metabolites, are the most critical GO terms, which are involved in the occurrence and development of AAU. In addition, pathway analysis identified Staphylococcus aureus infection, chemokine signaling pathways, cytokine-to-cytokine receptor interactions and metabolic pathways as the most important pathways. The result of the analysis of the function and pathways of DEGs supported that there were marked changes in the immune and inflammatory reactions in HLA-B27-positive AAU patients compared with the controls. We observed the inflammatory reaction of activated mononuclear cells in this experiment by using the LPS as a stimulus. It was observed from the pathway analysis that Gram-negative bacterial infection-related pathways activated other downstream pathways, including cytokine-to-cytokine receptor interactions, chemokine signaling pathways, gap junctions, Fc $\gamma$ R-mediated phagocytosis and several diverse metabolic pathways.
To screen for the important genes, Signal-Net and Gene-Rel-Net analyses were performed and identified 18 genes that were selected as key genes. Amongst these, particular attention was paid to CDC42 and IRAK3/M, as our studies suggested that these two genes were more significant compared with others (Table I). CDC42 is a member of the Rho GTPase family that regulates the organization of the cytoskeleton and membrane, and is involved in cell proliferation, polarity and motility (33). The Rho family of small GTPases are critical factors involved in the regulation of signal transduction cascades from extracellular stimuli to the cell nucleus, including the JNK/SAPK signaling pathway (34). CDC42 has been demonstrated to be a signal point for intracellular signaling networks that monitor multiple signaling pathways, including cytokine receptors, integrins, and responses to physical and chemical stresses (35). Ito et al demonstrated that knockdown of the CDC42 pathway significantly inhibited the upregulation of inflammatory genes, decreased the increased levels of pro-inflammatory molecules, consequently attenuating the overactivation of immunity. $\mathrm{CDC} 42$ regulates 

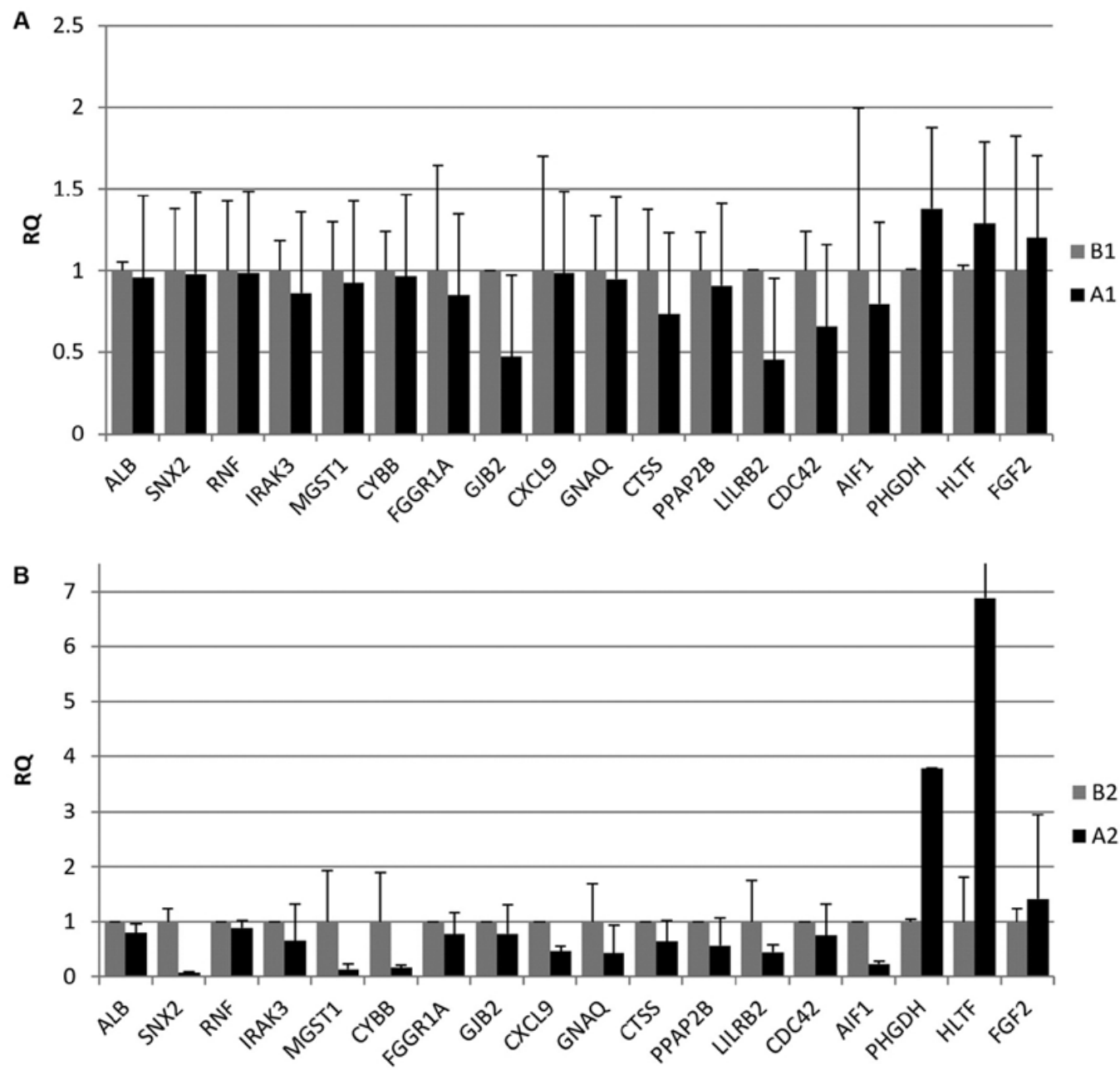

Figure 6. Reverse transcription-quantitative polymerase chain reaction (RT-qPCR) validation of 18 differentially expressed genes. (A) Difference between A1 and B1. (B) Difference between A2 and B2. Total RNA was extracted for RT-qPCR to analyze the expression of 18 important genes identified by Signal-Net analysis and Gene-Rel-Net. The results are shown as mean \pm standard deviation of three independent experiments $(P<0.05)$. The $y$-axis shows the relative mRNA expression and the $\mathrm{x}$-axis shows the 18 important genes.

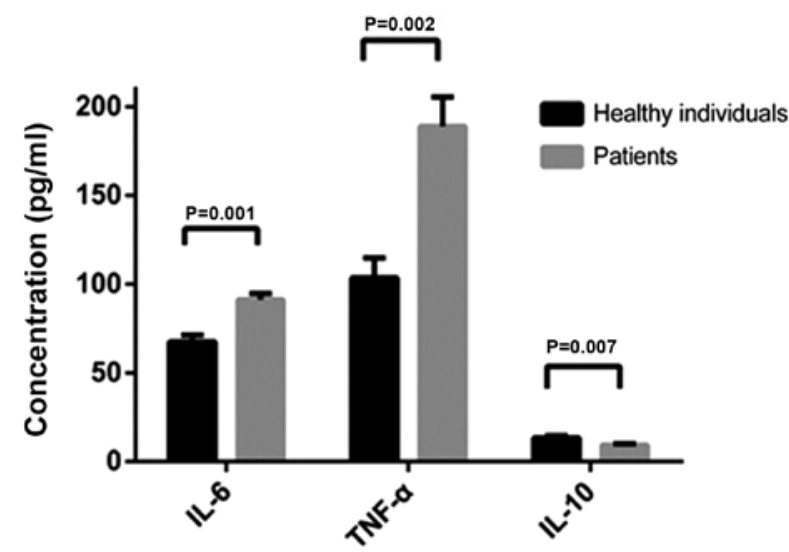

Figure 7. ELISA analysis of culture supernatants. The increased concentration of tumor necrosis factor (TNF)- $\alpha$, interleukin (IL)- 6 and IL-10 in the culture supernatants of monocytes isolated from the patients and their blood relatives folowing stimulation by lipopolysaccharides. Cytokine levels are expressed as mean \pm standard deviation (vertical bars) in $\mathrm{pg} / \mathrm{ml}$.

the expression of pro-inflammatory molecules by mobilizing the NF- $\mathrm{KB}$ pathway, which is closely associated with LPS-mediated TLR4 activation (34).
Due to the importance of the immune process, other key genes may be worth investigating. Interleukin-1 receptor-associated kinase M/3 (IRAKM/3), unlike other active members of the IRAK family (IRAK-1 and -4) is a negative regulator of TLR-mediated immune responses and it was downregulated in our results. The activation of IRAK-M/3 prevents the dissociation of IRAK-1 and -4 from MyD88 and formation of IRAK-TRAF6 complexes, thereby suppressing production of the downstream pro-inflammatory mediators controlled by TLR signaling (36-38). Moreover, it is worth noting that the IRAK-M/3 is crucial for endotoxin tolerance and its absence enhances the action of inflammatory cytokines (39). Overexpression of IRAK-M/3 can downregulate TLR signaling, thus playing an important role in controlling inflammatory and immune responses. In the present study, the expression of IRAK-M/3 was downregulated in monocytes of AAU patients following stimulation with LPS, as proven in an in vivo study conducted simultaneously. In this sense, we hypothesized that the reduction in IRAKM/3 expression was significantly correlated with HLA-B27-positive AAU patients.

In the present study, we also compared the concentrations of TNF- $\alpha$, IL-10 and IL- 6 in the culture supernatants of monocytes 
isolated from patients and their blood relatives. In our previous study, high levels of IL-6 were detected in the aqueous humor of $\mathrm{C} 3 \mathrm{H} / \mathrm{HeN}$ mice during endotoxin-induced uveitis (40). In addition, the concentrations of TNF- $\alpha$ and IL-10 in the culture supernatants of peripheral blood mononuclear cells isolated from patients with HLA-B27-associated AAU were found to be significantly increased following stimulation with LPS (41). In addition, the concentrations of IL- 6 , TNF- $\alpha$ and IL-10 were all found to be increased following LPS stimulation in the patients as well as their blood relatives. Subsequently, the elevated concentrations of IL-6, TNF- $\alpha$ and IL-10 in the culture supernatants of monocytes were compared between the patients and their blood relatives following LPS stimulation. Compared with the non-stimulatory group, the concentrations of IL- 6 and TNF- $\alpha$ in the patients were significantly higher in the LPS stimulation group. By contrast, the concentration of IL-10 cytokine was significantly higher in the blood relatives. To the best of our knowledge, the pro-inflammatory cytokines IL- 6 and TNF- $\alpha$ regulate various aspects of the immune response $(42,43)$, whereas IL-10 acts as an immunosuppressive cytokine $(44,45)$. Our results demonstrated that there were higher levels of inflammation and lower anti-inflammatory effects in patients with HLA-B27-associated AAU.

In conclusion, monocytes isolated from patients may produce a more intense inflammatory response compared with those isolated from their healthy blood relatives following stimulation with LPS, and monocytes isolated from patients were found to be more sensitive to LPS stimulation. This may be a possible explanation as to why patients with HLA-B27-associated AAU and their healthy HLA-B27-positive blood relatives are all seropositive for HLA-B27, but only the former suffer from AAU. Using gene chip technology to compare monocytes from HLA-B27-positive families with AAU with the control group following LPS stimulation, we identified several potential target genes. Experiments on the peripheral blood of humans and animals are currently being conducted to further elucidate the roles of these key genes in the development of HLA-B27-positive AAU. Future investigation into differentially expressed genes should provide insights into the pathogenesis of AAU.

\section{Acknowledgements}

The authors would like to thank Professor Guilin Xie (Lanzhou Institute of Biological Products) for providing LPS.

\section{Funding}

This study was funded by the National Natural Science Foundation of China under grant nos. 81273246 and 81471575.

\section{Availability of data and materials}

The analyzed data sets generated during the study are available from the corresponding author on reasonable request.

\section{Authors' contributions}

SY and CM participated in designing the study, performed part of the experiments, analyzed the patient data, drafted and revised the manuscript. JY performed part of the experiments.
XQ recruited the patients. JW participated in designing the study. HL participated in designing the study, recruited some of the patients and revised the manuscript. All authors read and approved the final manuscript.

\section{Ethics approval and consent to participate}

All candidates provided written informed consent and the clinical research protocol was approved by the Ethics Committee of Beijing Chao-Yang Hospital, Capital Medical University.

\section{Consent for publication}

Not applicable.

\section{Competing interests}

The authors declare that they have no competing interests.

\section{References}

1. Muñoz-Fernández S and Martín-Mola E: Uveitis. Best Pract Res Clin Rheumatol 20: 487-505, 2006.

2. Jabs DA, Nussenblatt RB and Rosenbaum JT; Standardization of Uveitis Nomenclature (SUN) Working Group: Standardization of uveitis nomenclature for reporting clinical data. Results of the First International Workshop. Am J Ophthalmol 140: 509-516, 2005.

3. Wakefield D, Yates W, Amjadi S and McCluskey P: HLA-B27 anterior uveitis: Immunology and immunopathology. Ocul Immunol Inflamm 24: 450-459, 2016.

4. McGonagle D and McDermott MF: A proposed classification of the immunological diseases. PLoS Med 3: e297, 2006.

5. Smith JA, Märker-Hermann E and Colbert RA: Pathogenesis of ankylosing spondylitis: Current concepts. Best Pract Res Clin Rheumatol 20: 571-591, 2006.

6. Colbert RA: HLA-B27 misfolding: A solution to the spondyloarthropathy conundrum? Mol Med Today 6: 224-230, 2000.

7. Schröder M and Kaufman RJ: ER stress and the unfolded protein response. Mutat Res 569: 29-63, 2005.

8. Chang JH, McCluskey PJ and Wakefield D: Acute anterior uveitis and HLA-B27. Surv Ophthalmol 50: 364-388, 2005.

9. Goldman M: Translational mini-review series on Toll-like receptors: Toll-like receptor ligands as novel pharmaceuticals for allergic disorders. Clin Exp Immunol 147: 208-216, 2007.

10. Du L, Kijlstra A and Yang P: Immune response genes in uveitis. Ocul Immunol Inflamm 17: 249-256, 2009.

11. Bi HS, Liu ZF and Cui Y: Pathogenesis of innate immunity and adaptive immunity in the mouse model of experimental autoimmune uveitis. J Chin Med Assoc 78: 276-282, 2015.

12. Hu XF, Lu H, Wang J, Zhang XS, Zhang XL, Liu XH, Xu ZZ, $\mathrm{Hu}$ JM and Lu QJ: Screening of key genes and inflammatory signalling pathway involved in the pathogenesis of HLA-B27associated acute anterior uveitis by gene expression microarray. Zhonghua Yan Ke Za Zhi 49: 217-223, 2013 (In Chinese).

13. Deschenes J, Murray PI, Rao NA, Nussenblatt RB; International Uveitis Study Group: International Uveitis Study Group (IUSG): clinical classification of uveitis. Ocul Immunol Inflamm 16:1-2, 2008.

14. Wright GW and Simon RM: A random variance model for detection of differential gene expression in small microarray experiments. Bioinformatics 19: 2448-2455, 2003.

15. Yang H, Crawford N, Lukes L, Finney R, Lancaster M and Hunter KW: Metastasis predictive signature profiles pre-exist in normal tissues. Clin Exp Metastasis 22: 593-603, 2005.

16. Clarke R, Ressom HW, Wang A, Xuan J, Liu MC, Gehan EA and Wang Y: The properties of high-dimensional data spaces: Implications for exploring gene and protein expression data. Nat Rev Cancer 8: 37-49, 2008.

17. Dupuy D, Bertin N, Hidalgo CA, Venkatesan K, Tu D, Lee D, Rosenberg J, Svrzikapa N, Blanc A, Carnec A, et al: Genome-scale analysis of in vivo spatiotemporal promoter activity in Caenorhabditis elegans. Nat Biotechnol 25: 663-668, 2007. 
18. Gene Ontology Consortium: The Gene Ontology (GO) project in 2006. Nucleic Acids Res 34: D322-D326, 2006.

19. Ashburner M, Ball CA, Blake JA, Botstein D, Butler $\mathrm{H}$ Cherry JM, Davis AP, Dolinski K, Dwight SS, Eppig JT, et al; The Gene Ontology Consortium: Gene ontology: Tool for the unification of biology. Nat Genet 25: 25-29, 2000.

20. Wei $\mathrm{Z}$ and $\mathrm{Li} \mathrm{H}$ : A Markov random field model for network-based analysis of genomic data. Bioinformatics 23: 1537-1544, 2007.

21. Li C and $\mathrm{Li} \mathrm{H}$ : Network-constrained regularization and variable selection for analysis of genomic data. Bioinformatics 24 1175-1182, 2008.

22. Zhang JD and Wiemann S: KEGGgraph: A graph approach to KEGG PATHWAY in R and bioconductor. Bioinformatics 25 : 1470-1471, 2009.

23. Pujana MA, Han JD, Starita LM, Stevens KN, Tewari M, Ahn JS Rennert G, Moreno V, Kirchhoff T, Gold B, et al: Network modeling links breast cancer susceptibility and centrosome dysfunction. Nat Genet 39: 1338-1349, 2007.

24. Prieto C, Risueño A, Fontanillo C and De las Rivas J: Human gene coexpression landscape: Confident network derived from tissue transcriptomic profiles. PLoS One 3: e3911, 2008.

25. Chang JH and Wakefield D: Uveitis: A global perspective. Ocul Immunol Inflamm 10: 263-279, 2002.

26. Bhattacherjee P, Williams RN and Eakins KE: An evaluation of ocular inflammation following the injection of bacterial endotoxin into the rat foot pad. Invest Ophthalmol Vis Sci 24: 196-202, 1983

27. McMenamin PG and Crewe J: Endotoxin-induced uveitis Kinetics and phenotype of the inflammatory cell infiltrate and the response of the resident tissue macrophages and dendritic cells in the iris and ciliary body. Invest Ophthalmol Vis Sci 36: 1949-1959, 1995.

28. de Vos AF, van Haren MA, Verhagen C, Hoekzema R and Kijlstra A: Kinetics of intraocular tumor necrosis factor and interleukin-6 in endotoxin-induced uveitis in the rat. Invest Ophthalmol Vis Sci 35: 1100-1106, 1994.

29. Zheng C, Lei C, Chen Z, Zheng S, Yang H, Qiu Y and Lei B: Topical administration of diminazene aceturate decreases inflammation in endotoxin-induced uveitis. Mol Vis 21: 403-411, 2015.

30. Chen W, Hu X, Zhao L, Li S and Lu H: Expression of toll-like receptor 4 in uvea-resident tissue macrophages during endotoxininduced uveitis. Mol Vis 15: 619-628, 2009.

31. Li S, Lu H, Hu X, Chen W, Xu Y and Wang J: Expression of TLR4-MyD88 and NF- $\mathrm{KB}$ in the iris during endotoxin-induced uveitis. Mediators Inflamm 2010: 748218-748218, 2010.

32. Wakefield D, Chang JH, Amjadi S, Maconochie Z, Abu El-Asrar A and McCluskey P: What is new HLA-B27 acute anterior uveitis? Ocul Immunol Inflamm 19: 139-144, 2011.

33. Ito TK, Yokoyama M, Yoshida Y, Nojima A, Kassai H, Oishi K, Okada S, Kinoshita D, Kobayashi Y, Fruttiger M, et al: A crucial role for CDC42 in senescence-associated inflammation and atherosclerosis. PLoS One 9: e102186, 2014.
34. Perona R, Montaner S, Saniger L, Sánchez-Pérez I, Bravo R and Lacal JC: Activation of the nuclear factor-kappaB by Rho, CDC42, and Rac-1 proteins. Genes Dev 11: 463-475, 1997.

35. Puls A, Eliopoulos AG, Nobes CD, Bridges T, Young LS and Hall A: Activation of the small GTPase Cdc42 by the inflammatory cytokines TNF(alpha) and IL-1, and by the Epstein-Barr virus transforming protein LMP1. J Cell Sci 112: 2983-2992, 1999.

36. Kobayashi K, Hernandez LD, Galán JE, Janeway CA Jr, Medzhitov R and Flavell RA: IRAK-M is a negative regulator of Toll-like receptor signaling. Cell 110: 191-202, 2002.

37. Nakayama K, Okugawa S, Yanagimoto S, Kitazawa T, Tsukada K, Kawada M, Kimura S, Hirai K, Takagaki Y and Ota Y: Involvement of IRAK-M in peptidoglycan-induced tolerance in macrophages. J Biol Chem 279: 6629-6634, 2004.

38. Gottipati S, Rao NL and Fung-Leung WP: IRAK1: A critical signaling mediator of innate immunity. Cell Signal 20: 269-276, 2008.

39. Maldifassi MC, Atienza G, Arnalich F, López-Collazo E, Cedillo JL, Martín-Sánchez C, Bordas A, Renart J and Montiel C: A new IRAK-M-mediated mechanism implicated in the antiinflammatory effect of nicotine via $\alpha 7$ nicotinic receptors in human macrophages. PLoS One 9: e108397, 2014.

40. Xu Y, Chen W, Lu H, Hu X, Li S, Wang J and Zhao L: The expression of cytokines in the aqueous humor and serum during endotoxin-induced uveitis in $\mathrm{C} 3 \mathrm{H} / \mathrm{HeN}$ mice. Mol Vis 16 : 1689-1695, 2010.

41. Liu X, Hu X, Zhang X, Li Z and Lu H: Role of rheum polysaccharide in the cytokines produced by peripheral blood monocytes in TLR4 mediated HLA-B27 associated AAU. Biomed Res Int 2013: 431232, 2013.

42. Santos Lacomba M, Marcos Martín C, Gallardo Galera JM, Gómez Vidal MA, Collantes Estévez E, Ramírez Chamond R and Omar M: Aqueous humor and serum tumor necrosis factoralpha in clinical uveitis. Ophthalmic Res 33: 251-255, 2001.

43. Pérez-Guijo V, Santos-Lacomba M, Sánchez-Hernández M, Castro-Villegas MC, Gallardo-Galera JM and CollantesEstévez E: Tumour necrosis factor-alpha levels in aqueous humour and serum from patients with uveitis: The involvement of HLA-B27. Curr Med Res Opin 20: 155-157, 2004.

44. Mosmann TR: Properties and functions of interleukin-10. Adv Immunol 56: 1-26, 1994.

45. Groux H, Bigler M, de Vries JE and Roncarolo MG: Inhibitory and stimulatory effects of IL-10 on human $\mathrm{CD}^{+} \mathrm{T}$ cells. J Immunol 160: 3188-3193, 1998.

This work is licensed under a Creative Commons Attribution-NonCommercial-NoDerivatives 4.0 International (CC BY-NC-ND 4.0) License. 